\title{
Correlation between the protein expression of A-kinase anchor protein 95, cyclin D3 and AKT and pathological indicators in lung cancer tissues
}

\author{
XIUYI YU ${ }^{1}$, YANGYANG YUAN ${ }^{2}$, XUEHONG ZHI ${ }^{2}$, BOGANG TENG ${ }^{2}$, XIAOXUAN CHEN $^{2}$, \\ QIAN HUANG ${ }^{2}$, YUEXIN CHEN ${ }^{2}$, ZHIYU GUAN ${ }^{3}$ and YONGXING ZHANG ${ }^{2}$
}

\begin{abstract}
${ }^{1}$ First Affiliated Hospital of Xiamen University; ${ }^{2}$ State Key Laboratory of Molecular Vaccinology and Molecular Diagnostics, School of Public Health, Xiamen University, Xiamen, Fujian 361102; ${ }^{3}$ Department of Cardiovascular and Thoracic Surgery, Tianjin Medical University General Hospital, Tianjin 300052, P.R. China
\end{abstract}

Received June 22, 2014; Accepted February 13, 2015

DOI: $10.3892 /$ etm.2015.2637

\begin{abstract}
The aim of the present study was to investigate the correlation between the protein expression of A-kinase anchor protein 95 (AKAP95), cyclin D3 and AKT with pathological indicators in lung cancer tissues. Immunohistochemistry was used to detect the protein expression levels of the proteins in 51 lung cancer tissue samples and 15 pericarcinoma tissue samples. The percentage of cyclin D3 positive samples in the lung cancer and pericarcinoma tissues was 68.63 and $28.57 \%$, respectively, and the difference was statistically significant $(\mathrm{P}<0.01)$. However, cyclin D3 expression was not shown to correlate with differentiation grade, histological type or lymph node metastasis. In addition, the percentage of AKT positive samples in the cancer and pericarcinoma tissues was 76.47 and $38.46 \%$, respectively, and the difference was statistically significant $(\mathrm{P}<0.05)$. AKT expression was found to significantly correlate with the grade of cancer tissue differentiation $(\mathrm{P}<0.05)$; however, no correlations were observed with histological type or lymph node metastasis ( $>>0.05)$. AKAP95 expression was shown to correlate with cyclin D3 and AKT expression in the lung cancer tissue $(\mathrm{P}<0.05)$; however, there was no correlation between cyclin D3 and AKT expression. The present study provided evidence suggesting that AKAP95 may have a role in regulation of the cell cycle.
\end{abstract}

Correspondence to: Dr Yongxing Zhang, State Key Laboratory of Molecular Vaccinology and Molecular Diagnostics, School of Public Health, Xiamen University, 442 Simingnan Road, Xiamen, Fujian 361102, P.R. China

E-mail: z63y94x@xmu.edu.cn

Dr Zhiyu Guan, Department of Cardiovascular and Thoracic Surgery, Tianjin Medical University General Hospital, 154 Anshan Road, Tianjin 300052, P.R. China

E-mail: zhongns@thepbpc.org

Key words: lung cancer, A-kinase anchor protein 95, cyclin D3, AKT, diagnosis, biomarkers, pathology

\section{Introduction}

A-kinase anchor protein 95 (AKAP95) binds to the RII subunit of protein kinase A (PKA) and catalyzes the phosphorylation of target proteins; thus, ensuring the signal transduction process of the cyclic adenosine monophosphate (cAMP) pathway (1). AKAP95 has been found to strongly interact with all D-type cyclins and regulate cyclin D3-cyclin-dependent kinase (CDK)4 activity in cotransfected Chinese hamster ovary cells (2). Cyclin D/E is able to form a cyclin D/E-AKAP95-PKA complex with the RII subunit of PKA via the AKAP95 protein during the G1/S phase to subsequently promote cell proliferation (3). Cyclin D3, a subtype of D-type cyclins, has been reported to be overexpressed in non-Hodgkin lymphoma and primary invasive breast cancer $(4,5)$. AKT, a proteohormone with multiple functions, is not only involved in cell growth, metabolism and apoptosis, but is also associated with the initiation and development of cancer. A previous study reported that AKT activated CDK4 through regulating the expression of cyclin D1, cyclin D2, p21 and p57 in the G1 phase, and subsequently promoting pancreatic $\beta$-cell proliferation in mice (6). Therefore, it was hypothesized that correlations may exist between the expression of AKAP95, cyclin D3 and AKT. In the present study, the correlations among these three proteins and their association with pathology indicators was investigated by analyzing the expression of these proteins in 51 lung cancer tissue samples.

\section{Materials and methods}

Sample collection. A total of 51 lung cancer tissue samples were collected surgically from lung cancer patients, aged

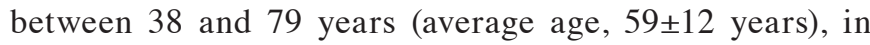
Shengjing Hospital of China Medical University (Shenyang, China) between 2007 and 2009. The specimens were pathologically diagnosed by pathology experts, and were grouped according to the following diagnostic results: Differentiation grade, histological type and presence of lymph node metastasis. Furthermore, 15 samples of pericarcinoma tissue, which were located $3 \mathrm{~cm}$ away from the cancerous tissue and did 
not contain any cancer cells (pathologically identified), were used as a control. The present study was approved by the ethics committee of Xiamen University (Xiamen, China), and written informed consent was obtained from the patients or their families.

Reagents and immunohistochemistry (IHC). Specimens were cut into $4-\mu \mathrm{m}$ sections, fixed with $10 \%$ neutral-buffered formalin and embedded in paraffin. A streptavidin-peroxidase (SP) IHC method was applied. The SP IHC kit, 3,3'-diaminobenzidine substrate and hematoxylin were obtained from Maxim Biotechnology Co., Ltd (Fuzhou, China). Hematoxylin was used to stain the tissues. Mouse anti-human primary monoclonal antibodies against AKAP95 (1:150; cat. no. sc-100643) and cyclin D3 (1:300; cat. no. sc-6283) were obtained from Santa Cruz Biotechnology, Inc. (Dallas, TX, USA). Mouse monoclonal anti-AKT antibody (1:150; cat. no. 2920S) was purchased from Cell Signaling Technology, Inc. (Danvers, MA, USA). Phosphate-buffered saline was used as a negative control for the antibody.

Criteria for analysis of the IHC results. When the slides were observed through a microscope (BA310Digital; Motic China Group Co., Ltd, Xiamen, China), positive protein expression was indicated by a yellow-brown color, which was caused by the protein immunoreaction. By contrast, protein expression was considered to be negative when no yellow-brown color was observed. For each slide, 10 fields of view were randomly selected and observed, and in each field of view, 200 cancer cells were counted and the protein expression was observed. The percentage of positive cells was calculated and defined as shown in Table I. In the statistical analysis, - and +- were considered to indicate negative protein expression, while,+++ and +++ were considered to indicate positive protein expression (7).

Statistical analysis. Statistical analysis was performed using SPSS software (version 13.0; SPSS, Inc., Chicago, IL, USA). The constituent ratio of subjects was compared with the $\chi 2$ test, while correlations were analyzed using Spearman's rank correlation analysis. $\mathrm{P}<0.05$ ( $\alpha$ level) was considered to indicate a statistically significant difference.

\section{Results}

Expression of AKAP95, cyclin D3 and AKT in lung cancer and pericarcinoma tissues. Expression levels of AKAP95 in the lung cancer and pericarcinoma tissues were reported as follows: The percentage of AKAP95 positive samples was $82.35 \%$ in the cancer tissue and $33.33 \%$ in the pericarcinoma tissue. The difference between them was statistically significant $(\mathrm{P}<0.01)$, as shown in Table II.

Expression of cyclin D3 in lung cancer and pericarcinoma tissues. Positive expression of cyclin D3 was $68.63 \%(35 / 51)$ in the cancer tissues and $28.57 \%$ (4/14) in the pericarcinoma tissue. As shown in Table III, the difference in the positive rate of cyclin D3 expression between the cancer and pericarcinoma tissues was statistically significant $(\mathrm{P}<0.01)$. The protein expression of cyclin D3 is shown in Fig. 1.
Table I. Criteria for the analysis of immunohistochemical results.

\begin{tabular}{lr} 
Range & Positivity \\
\hline $0-<10 \%$ & - \\
$\geq 10 \%,<25 \%$ & +- \\
$\geq 25 \%,<50 \%$ & + \\
$\geq 50 \%,<75 \%$ & ++ \\
$\geq 75 \%$ & +++ \\
\hline
\end{tabular}

Expression of AKT in lung cancer and pericarcinoma tissues. Positive expression of AKT, as shown in Fig. 2, in the cancer and pericarcinoma tissues was $76.47 \%(39 / 51)$ and $38.46 \%$ (5/13), respectively. As shown in Table IV, the difference in the positive ratio of AKT expression between the cancer tissue and pericarcinoma tissue was statistically significant $(\mathrm{P}<0.05)$.

Correlations between the expression of AKAP95, cyclin D3 and AKT with pathology indicators in lung cancer tissue. Correlations between the expression of AKAP95, cyclin D3 and AKT with pathology indicators are shown in Table V. AKAP95 expression was shown to correlate with the grade of cancer tissue differentiation and histological type, but not with lymph node metastasis. No statistically significant differences were observed in the cyclin D3 expression levels in cancer tissues with different grades of differentiation, tissues with different histological type or tissues with or without lymph node metastasis $(\mathrm{P}>0.05)$, indicating that cyclin D3 expression was not correlated with differentiation grade, histological type or lymph node metastasis (Table V). With regard to AKT expression, a statistically significant difference was observed between tissues with different grades of differentiation $(\mathrm{P}<0.05)$; however, no statistically significant differences were observed in between the tissues with a different histological type or in tissues with or without lymph node metastasis ( $\mathrm{P}>0.05)$, which indicated that AKT expression was correlated with the grade of differentiation, but not with the histological type or lymph node metastasis.

Correlations among AKAP95, cyclin D3 and AKT expression levels in lung cancer tissue. As shown in Tables VI and VII, AKAP95 expression was found to significantly correlate with cyclin D3 and AKT expression $(\mathrm{P}<0.05)$. However, no statistically significant correlation was observed between cyclin D3 and AKT expression ( $\mathrm{P}>0.05$; Table VIII).

\section{Discussion}

With the rapid development of society and the increasing rate of industrialization, individuals are exposed to more chemical carcinogens. Investigating the molecular mechanisms underlying lung cancer development and progression is vital. AKAP95 is a protein belonging to the AKAP family that is located in the nucleus. As an anchoring protein to PKA, AKAP95 binds to PKA through a PKA RII subunit, and is subsequently involved in target protein phosphorylation by 
Table II. AKAP95 expression in lung cancer and pericarcinoma tissues.

\begin{tabular}{lcccc}
\hline AKAP95 & Pericarcinoma & Lung cancer & $\chi^{2}$ & P-value \\
\hline Positive & 5 & 42 & 13.59 & 0.001 \\
Negative & 10 & 9 &
\end{tabular}

A statistically significant difference in the positivity ratio of AKAP95 expression was observed between the lung cancer and pericarcinoma tissues. AKAP, A-kinase anchor protein.

Table III. Cyclin D3 expression in lung cancer and pericarcinoma tissues.

\begin{tabular}{lcccc}
\hline Cyclin D3 & Pericarcinoma & Lung cancer & $\chi^{2}$ & P-value \\
\hline Positive & 4 & 35 & 7.344 & 0.007 \\
Negative & 10 & 16 & \\
\hline
\end{tabular}

A statistically significant difference in the positivity ratio of cyclin D3 expression was observed between the lung cancer and pericarcinoma tissues.

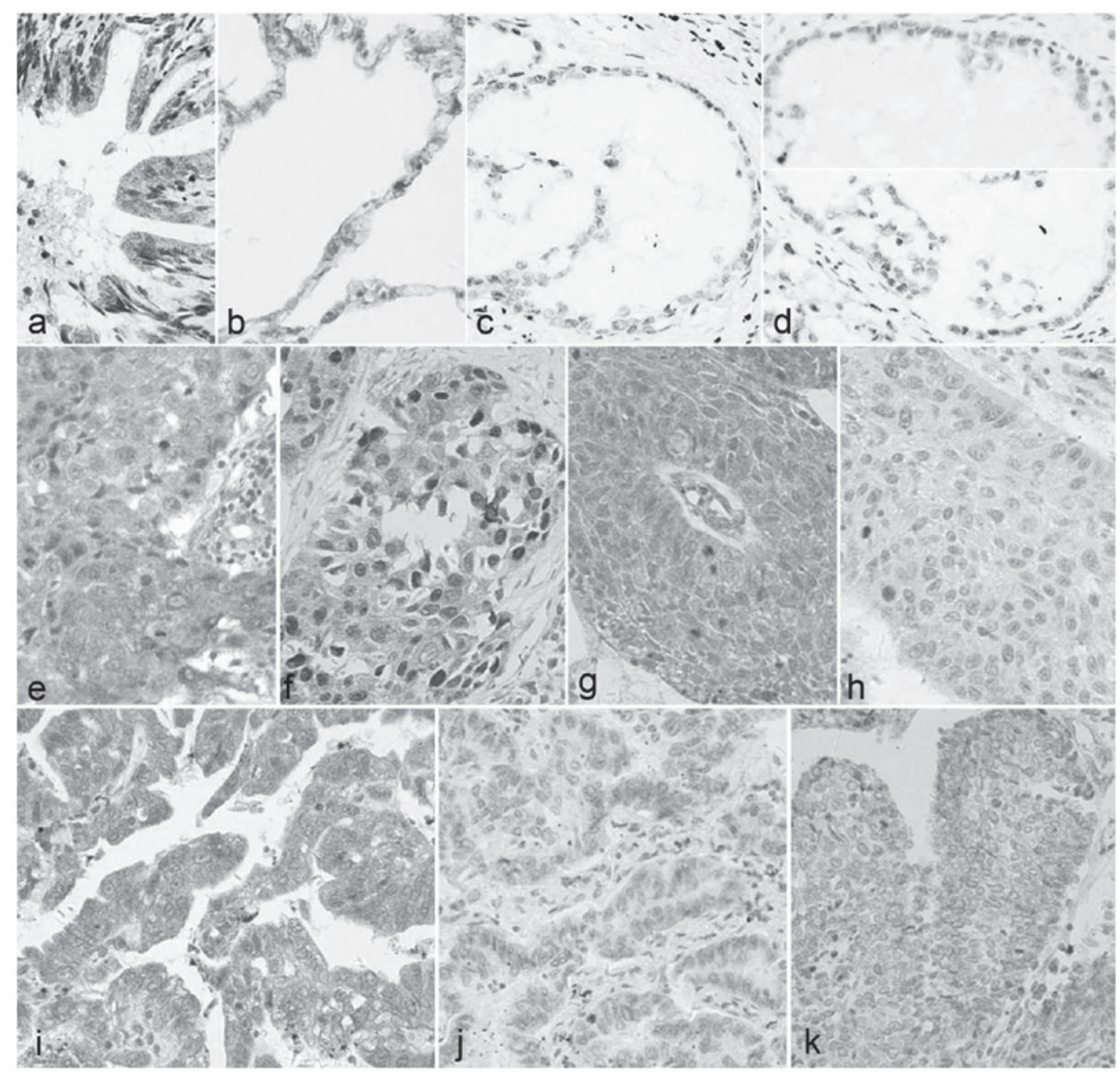

Figure 1. Expression of cyclin D3 was determined in pericarcinoma and lung cancer tissues using a streptavidin-peroxidase immunohistochemistry method (magnification, $\mathrm{x} 400$ ). (a and b) Positive expression of cyclin D3 in pericarcinoma tissue. Cyclin D3 was predominantly cytoplasmically localized and was expressed in the nucleus in a small number of cells. (c and d) Negative expression of cyclin D3 in pericarcinoma tissue, e.g. cyclin D3 was highly expressed in cytoplasm of cells from squamous cell lung carcinoma. (e and f) Cyclin D3 was highly expressed in the nucleus of cells from squamous cell lung carcinoma. ( $\mathrm{g}$ and $\mathrm{h}$ ) Low cyclin D3 expression was observed in the cytoplasm of cells from squamous cell lung carcinoma. (i) Cyclin D3 was highly expressed in the cytoplasm of cells from lung adenocarcinoma. (j) Low cyclin D3 expression was observed in the cytoplasm of cells from lung adenocarcinoma. (k) No cyclin D3 expression was observed in cells from small-cell lung cancer. 
Table IV. AKT expression in lung cancer and pericarcinoma tissues.

\begin{tabular}{lcccc}
\hline AKT & Pericarcinoma & Lung cancer & $\chi^{2}$ & P-value \\
\hline Positive & 5 & 39 & 5.309 & 0.021 \\
Negative & 8 & 12 &
\end{tabular}

A statistically significant difference in the positivity ratio of AKT expression was observed between the lung cancer and pericarcinoma tissues.

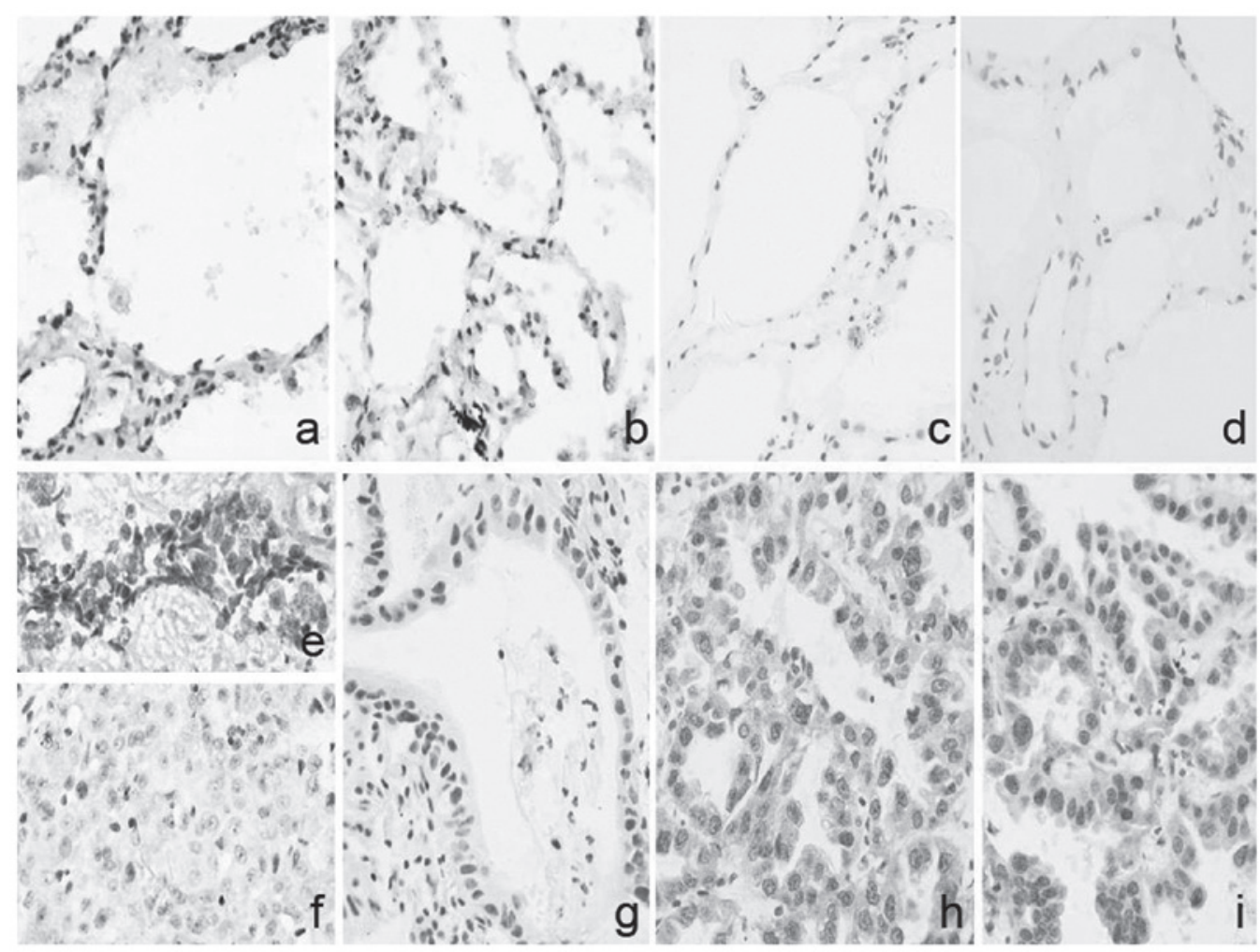

Figure 2. Expression of AKT was determined in lung pericarcinoma and cancer tissues using a streptavidin-peroxidase immunohistochemistry method (magnification, x400). (a and b) Positive expression of AKT in the pericarcinoma lung tissue, localized in the nucleus. (c and d) Negative expression of AKT in the pericarcinoma lung tissue. (e) Positive expression of AKT in cells from squamous cell lung carcinoma, localized predominantly in the cytoplasm and nucleus. (f) Low expression of AKT was observed in the cytoplasm of cells from squamous cell lung carcinoma. (g) Low expression of AKT was observed in the nucleus of cells from squamous cell lung carcinoma. (h and i) High expression of AKT was observed in the nucleus of cells from lung adenocarcinoma.

PKA and cAMP signal transduction (1). AKAP95 also plays an important role in chromosome condensation in mitosis, DNA replication and cell apoptosis (8-11), and is able to regulate cyclin D3-CDK4 activity. Phosphoinositide 3-kinase (PI3K), an activator of AKT protein (12), has a synergistic effect with cyclin D3 in humans and mice, on promoting Burkitt lymphoma (13). AKT phosphorylates the Ser9 of glycogen synthase kinase (GSK) $3 \beta$ and further upregulates cyclin D1 and cyclin D2 expression, but not cyclin D3 expression (6). Thus, the present study investigated the expression of AKAP95, cyclin D3 and AKT and the correlations among these three proteins.

Abnormal cell cycle regulation during the G1/S phase is critical in tumor development. Type D cyclins contain three members, namely cyclins D1, D2 and D3. Cyclins D1 and D2 have been confirmed to be overexpressed in multiple types of tumor, indicating their potential as oncogenes $(14,15)$. Out of 198 cases of non-Hodgkin lymphoma, 43 cases exhibited cyclin D3 overexpression (21.72\%), while out of 170 cases of primary invasive breast cancer 38 cases exhibited cyclin D3 overexpression $(22.36 \%)(4,5)$. A previous study reported that cyclin D3 expression was positive in $23 / 33$ cases of primary lung cancer (69.70\%) (16). The current study demonstrated that the positivity ratio of cyclin D3 expression in lung cancer tissue was $68.63 \%$, which was significantly higher compared with that in pericarcinoma tissue $(28.57 \%)$. In addition, the positivity ratio of cyclin D3 expression in lung cancer was higher compared with that in non-Hodgkin lymphoma and primary invasive breast cancer, which may be associated with the histological type of the tumor. Cyclin D3 expression in non-Hodgkin lymphoma was found to correlate with the clinical groupings (4). In the present study, cyclin D3 expression was not shown to correlate with the grade of cancer tissue differentiation, histological type or lymph node metastasis, while cyclin D1 expression has been previously correlated with histological type, but not with the grade of cancer tissue 
Table V. Associations between the protein expression of AKAP95, cyclin D3 and AKT with the pathology index in lung cancer tissues.

\section{A, AKAP95}

\begin{tabular}{|c|c|c|c|c|c|}
\hline Item & Cases (n) & Positive (n) & Negative (n) & $\chi^{2}$ & P-value \\
\hline Grade of differentiation & & & & & 0.029 \\
\hline Well-differentiated & 4 & 2 & 2 & 6.49 & \\
\hline Moderately differentiated & 25 & 24 & 1 & & \\
\hline Poorly differentiated & 19 & 15 & 4 & & \\
\hline Histological type & & & & & 0.018 \\
\hline Small-cell lung cancer & 9 & 7 & 2 & 9.00 & \\
\hline Squamous cell lung carcinoma & 22 & 20 & 2 & & \\
\hline Lung adenocarcinoma & 15 & 14 & 1 & & \\
\hline Alveolar carcinoma & 4 & 1 & 3 & & \\
\hline Lymph node & & & & & 0.714 \\
\hline Positive & 29 & 23 & 6 & 0.43 & \\
\hline Negative & 22 & 19 & 3 & & \\
\hline
\end{tabular}

B, Cyclin D3

\begin{tabular}{|c|c|c|c|c|c|}
\hline Item & Cases (n) & Positive (n) & Negative (n) & $\chi^{2}$ & P-value \\
\hline Grade of differentiation & & & & & 0.076 \\
\hline Well-differentiated & 4 & 3 & 1 & 5.208 & \\
\hline Moderately differentiated & 25 & 22 & 3 & & \\
\hline Poorly differentiated & 19 & 11 & 8 & & \\
\hline Histological type & & & & & 0.063 \\
\hline Small-cell lung cancer & 9 & 3 & 6 & 6.715 & \\
\hline Squamous cell lung carcinoma & 22 & 18 & 4 & & \\
\hline Lung adenocarcinoma & 15 & 11 & 4 & & \\
\hline Alveolar carcinoma & 4 & 3 & 1 & & \\
\hline Lymph node & & & & & 0.583 \\
\hline Positive & 29 & 19 & 10 & 0.302 & \\
\hline Negative & 22 & 16 & 6 & & \\
\hline
\end{tabular}

$\mathrm{C}, \mathrm{AKT}$

\begin{tabular}{|c|c|c|c|c|c|}
\hline Item & Cases (n) & Positive (n) & Negative (n) & $\chi^{2}$ & P-value \\
\hline Grade of differentiation & & & & & 0.046 \\
\hline Well-differentiated & 4 & 1 & 3 & 5.542 & \\
\hline Moderately differentiated & 25 & 21 & 4 & & \\
\hline Poorly differentiated & 19 & 14 & 5 & & \\
\hline Histological type & & & & & 0.723 \\
\hline Small-cell lung cancer & 9 & 7 & 2 & 4.459 & \\
\hline Squamous cell lung carcinoma & 22 & 14 & 8 & & \\
\hline Lung adenocarcinoma & 15 & 14 & 1 & & \\
\hline Alveolar carcinoma & 4 & 3 & 1 & & \\
\hline Lymph node & & & & & 0.906 \\
\hline Positive & 29 & 22 & 7 & 0.014 & \\
\hline Negative & 22 & 17 & 5 & & \\
\hline
\end{tabular}

AKAP95, A-kinase anchor protein 95. 
Table VI. Correlation analysis between AKAP95 and cyclin D3 expression.

\begin{tabular}{|c|c|c|c|c|c|c|c|}
\hline \multirow[b]{2}{*}{ AKAP95 } & \multicolumn{5}{|c|}{ Cyclin D3 } & \multirow[b]{2}{*}{ rs } & \multirow[b]{2}{*}{ P-value } \\
\hline & - & +- & + & ++ & +++ & & \\
\hline- & 1 & 1 & 1 & 0 & 0 & & \\
\hline+- & 0 & 2 & 1 & 0 & 1 & & \\
\hline+ & 1 & 1 & 3 & 1 & 0 & 0.346 & 0.013 \\
\hline++ & 3 & 5 & 9 & 5 & 2 & & \\
\hline+++ & 0 & 2 & 4 & 5 & 3 & & \\
\hline
\end{tabular}

AKAP95 expression was found to correlate with cyclin D3 expression in lung cancer tissue $(\mathrm{P}<0.05)$. rs, Spearman's rank correlation coefficient; AKAP, A-kinase anchor protein.

Table VII. Correlation analysis between AKAP95 and AKT expression.

\begin{tabular}{|c|c|c|c|c|c|c|c|}
\hline \multirow[b]{2}{*}{ AKAP95 } & \multicolumn{5}{|c|}{$\mathrm{AKT}$} & \multirow[b]{2}{*}{ rs } & \multirow[b]{2}{*}{$\mathrm{P}$-value } \\
\hline & - & +- & + & ++ & +++ & & \\
\hline- & 2 & 0 & 1 & 0 & 0 & & \\
\hline+- & 1 & 1 & 1 & 1 & 0 & & \\
\hline+ & 0 & 0 & 4 & 1 & 1 & 0.287 & 0.041 \\
\hline++ & 2 & 4 & 7 & 9 & 2 & & \\
\hline+++ & 0 & 2 & 4 & 6 & 2 & & \\
\hline
\end{tabular}

AKAP95 expression was found to correlate with AKT expression in lung cancer tissue $(\mathrm{P}<0.05)$. rs, Spearman's rank correlation coefficient; AKAP, A-kinase anchor protein.

Table VIII. Correlation analysis between cyclin D3 and AKT expression.

\begin{tabular}{|c|c|c|c|c|c|c|c|}
\hline \multirow[b]{2}{*}{ Cyclin D3 } & \multicolumn{5}{|c|}{$\mathrm{AKT}$} & \multirow[b]{2}{*}{ rs } & \multirow[b]{2}{*}{ P-value } \\
\hline & - & +- & + & ++ & +++ & & \\
\hline- & 1 & 1 & 1 & 2 & 0 & & \\
\hline+- & 1 & 2 & 3 & 2 & 3 & & \\
\hline+ & 3 & 3 & 8 & 4 & 0 & 0.215 & 0.130 \\
\hline++ & 0 & 0 & 4 & 7 & 0 & & \\
\hline+++ & 0 & 1 & 1 & 2 & 2 & & \\
\hline
\end{tabular}

Cyclin D3 expression was not found to correlate with AKT expression in lung cancer tissue (P>0.05). rs, Spearman's rank correlation coefficient.

differentiation or lymph node metastasis (17). Therefore, cyclin D3 may play a different role in different tumors, and the function of cyclin D1 and cyclin D3 may not be the same in the development of lung cancer, which is consistent with the different roles of cyclin D1 and cyclin D3 observed in gastric cancer development (18).

Overexpression of phosphorylated AKT is an independent prognostic factor of non-small-cell lung cancer (19). The present study revealed that the positivity ratio of AKT expression was $76.47 \%$ in lung cancer tissue, which was significantly higher compared with that in the pericarcinoma tissue $(38.46 \%)$.
AKT expression was also shown to correlate with the grade of cancer tissue differentiation, indicating the involvement of overexpression of AKT in lung cancer development. In an additional study by Mayadagli et al (12), the positivity ratio of AKT expression in squamous cell lung carcinoma was higher compared with that in non-squamous non-small cell lung cancer. Furthermore, in the current study, the positivity ratio of AKT expression in the different histological types was $93.3 \%$ (14/15) in lung adenocarcinoma, 77.8\% (7/9) in small cell lung cancer, $75 \%$ (3/4) in alveolar carcinoma and $63.64 \%(14 / 22)$ in squamous cell lung carcinoma. Thus, there is inconsistency 
with the study by Mayadagli et al, since the positivity ratio of AKT expression in squamous cell lung carcinoma was the lowest.

In contrast to the previously reported correlation with cyclin D1 expression in lung cancer (17), the present study demonstrated that AKAP95 expression was also correlated with cyclin D3 expression and AKT expression, indicating that AKAP95 may exert a synergistic effect on promoting tumor growth with AKT and cyclin D3. Abnormal activation of the oncogenic Ras protein has been shown to result in a strong activation of the PI3K/AKT pathway, as PI3K can activate AKT protein (12). This pathway is activated in the majority of cancer cells and plays critical roles in cell proliferation and apoptosis (20). Dominguez-Sola and Dalla-Favera (13) reported that PI3K and cyclin D3 exerted a synergistic effect on promoting Burkitt lymphoma, which possibly explains the synergistic effect of AKT with cyclin D3; however, AKT expression exhibited no correlation with cyclin D3 expression in the current study. AKT may upregulate cyclin D1 and cyclin D2 expression through phosphorylating the Ser9 of GSK3 $\beta$; however, is unable to upregulate cyclin D3 expression, which was consistent with the study by Fatrai et al (6).

In conclusion, the present study demonstrated that AKAP95 correlated with the expression of cyclin D3 and AKT in lung cancer tissues. In addition, AKT expression exhibited a strong correlation with the grade of cancer tissue differentiation; however, no correlation was observed with cyclin D3. The mechanism underlying the regulation of cyclin $\mathrm{D}$ proteins by AKT warrants further research. The present study identified a correlation between AKAP95, and both cyclin D3 and AKT. These results suggest that AKAP95 has an effect on the cell cycle through two different pathways.

\section{Acknowledgements}

This study was supported by grants from the National Natural Science Foundation (no. 81071927), the Fujian Innovation Project (no. 2012-CXB-25) and the Xiamen University Innovation Program (no. CXB2013024).

\section{References}

1. Diviani D, Dodge-Kafka KL, Li J and Kapiloff MS: A-kinase anchoring proteins: scaffolding proteins in the heart. Am J Physiol Heart Circ Physiol 301: H1742-H1753, 2011.

2. Arsenijevic T, Degraef C, Dumont JE, Roger PP and Pirson I: A novel partner for D-type cyclins: protein kinase A-anchoring protein AKAP95. Biochem J 378: 673-679, 2004

3. Arsenijevic T, Degraef C, Dumont JE, Roger PP and Pirson I: G1/S Cyclins interact with regulatory subunit of PKA via A-kinase anchoring protein, AKAP95. Cell Cycle 5: 1217-1222, 2006.
4. Møller MB, Nielsen O and Pedersen NT: Cyclin D3 expression in non-Hodgkin lymphoma. Correlation with other cell cycle regulators and clinical features. Am J Clin Pathol 115: 404-412, 2001.

5. Bukholm IR, Bukholm G and Nesland JM: Over-expression of cyclin A is highly associated with early relapse and reduced survival in patients with primary breast carcinomas. Int J Cancer 93: 283-287, 2001.

6. Fatrai S, Elghazi L, Balcazar N, et al: Akt induces beta-cell proliferation by regulating cyclin D1, cyclin D2 and p21 levels and cyclin-dependent kinase-4 activity. Diabetes 55: 318-325, 2006.

7. Hiscott PS, Grierson I and McLeod D: Retinal pigment epithelial cells in epiretinal membranes: an immunohistochemical study. Br J Ophthalmol 68: 708-715, 1984.

8. Collas P, Le Guellec K and Taskén K: The A-kinase-anchoring protein AKAP95 is a multivalent protein with a key role in chromatin condensation at mitosis. J Cell Biol 147: 1167-1180, 1999.

9. Steen RL, Cubizolles F, Le Guellec K and Collas P: A kinase-anchoring protein (AKAP)95 recruits human chromosome-associated protein (hCAP)-D2/Eg7 for chromosome condensation in mitotic extract. J Cell Biol 149: 531-536, 2000.

10. Eide T, Carlson C, Taskén KA, Hirano T, Taskén K and Collas P: Distinct but overlapping domains of AKAP95 are implicated in chromosome condensation and condensin targeting. EMBO Rep 3: 426-432, 2002.

11. Kamada S, Kikkawa U, Tsujimoto $\mathrm{Y}$ and Hunter T: A-kinase-anchoring protein 95 functions as a potential carrier for the nuclear translocation of active caspase 3 through an enzyme-substrate-like association. Mol Cell Biol 25: 9469-9477, 2005.

12. Mayadagli A, Karabulut Gul S, Bilici A, et al: Prognostic significance of protein kinase B/Akt pathway in patients with non-small cell lung cancer. J BUON 19: 157-163, 2014.

13. Dominguez-Sola D and Dalla-Favera R: Burkitt lymphoma: much more than MYC. Cancer Cell 22: 141-142, 2012.

14. Flørenes VA, Faye RS, Maelandsmo GM, Nesland JM and Holm R: Levels of cyclin D1 and D3 in malignant melanoma: deregulated cyclin D3 expression is associated with poor clinical outcome in superficial melanoma. Clin Cancer Res 6: 3614-3620, 2000.

15. Wolowiec D, Ciszak L, Kosmaczewska A, et al: Cell cycle regulatory proteins and apoptosis in B-cell chronic lymphocytic leukemia. Haematologica 86: 1296-1304, 2001.

16. Papay J, Krenacs T, Moldvay J, et al: Immunophenotypic profiling of nonsmall cell lung cancer progression using the tissue microarray approach. Appl Immunohistochem Mol Morphol 15: 19-30, 2007.

17. Hu SX, Kong XY, Yuan YY, et al: Relationship between AKAP95, cyclin E1, cyclin D1 and clinicopathological parameters in lung cancer tissue. Zhonghua Lao Dong Wei Sheng Zhi Ye Bing Za Zhi 31: 890-894, 2013 (In Chinese).

18. Yu J, Miehlke S, Ebert MP, et al: Expression of cyclin genes in human gastric cancer and in first degree relatives. Chin Med J (Engl) 115: 710-715, 2002.

19. David O, Jett J, LeBeau H, et al: Phospho-Akt overexpression in non-small cell lung cancer confers significant stage-independent survival disadvantage. Clin Cancer Res 10: 6865-6871, 2004

20. Osaki M, Oshimura M and Ito H: PI3K-Akt pathway: its functions and alterations in human cancer. Apoptosis 9: 667-676, 2004. 